\title{
Calidad editorial en revistas iberoamericanas de derecho y jurisprudencia desde la perspectiva de Latindex ${ }^{*}$
}

Editorial quality in Ibero-American law and jurisprudence journals from the Latindex perspective

\author{
Ana María Cetto** \\ José Octavio Alonso-Gamboa***
}

Resumen: Se presenta un análisis del perfil y la calidad editorial de 72 revistas iberoamericanas disponibles en línea, especializadas en derecho y jurisprudencia, que han ingresado al Catálogo 2.0 de Latindex. El catálogo acredita el cumplimiento de 38 características organizadas en cinco grupos, donde se evidencia el apego de estas revistas a diferentes normas y buenas prácticas editoriales. Los datos proporcionados permiten apreciar la importancia de contar con un sistema iberoamericano de información que es incluyente y recoge lo mejor de la producción editorial, sin caer en el elitismo o en el sesgo de los índices comerciales. El análisis presentado revela un panorama editorial dinámico en la región, en las disciplinas de derecho y jurisprudencia. El trabajo concluye con una reflexión sobre la razón de ser de las revistas del área jurídica en el contexto iberoamericano y los retos que enfrentan para seguir cumpliendo su función como vehículos para la comunicación científica.

Palabras clave: revistas académicas, calidad editorial, criterios de valoración, servicios de información, políticas de inclusión, Iberoamérica.

\footnotetext{
* Artículo de investigación Número Especial de Aniversario. Recibido el 30 de marzo de 2020 y aceptado para su publicación el 23 de julio de 2020.

** Profesora investigadora en el Instituto de Física, Universidad Nacional Autónoma de México. / ana@fisica.unam.mx / orcid.org/0000-0001-6006-1102

*** Académico titular en la Dirección General de Bibliotecas de la Universidad Nacional Autónoma de México. / oalonso@unam.mx /
orcid.org/0000-0002-0281-9613
} 
Abstract: We present an analysis of the editorial quality of 72 Ibero-American journals available online, specialized in Law and Jurisprudence, which are registered in the Latindex Catalogue 2.0. The Catalogue certifies compliance with 38 criteria, organized into five groups that account for the adhesion of a journal to a set of editorial norms and best practices. The data collected allow to appreciate the importance of relying on an Ibero-American information system that is inclusive and registers the best of the editorial production of the region, thus avoiding the elitism or bias of the commercial indexing services. The analysis presented reveals a vibrant editorial regional landscape in Law and Jurisprudence. We conclude with a reflection on the raison d'ettre of journals in this area of knowledge in the lbero-American context, and on the challenges faced by the journals in their efforts to continue serving as effective communication vehicles.

Keywords: scholarly journals, editorial quality, evaluation criteria, information services, inclusion policies, Ibero-America.

\section{Sumario}

1. Introducción

2. Las bases de datos y las revistas académicas

3. Las revistas de derecho y jurisprudencia en Latindex

4. La calidad editorial en las revistas de derecho y jurisprudencia

5. Reflexiones sobre el futuro de las revistas

\section{Introducción}

En las diferentes disciplinas del conocimiento, la revista académica continúa cumpliendo funciones esenciales para el proceso de comunicación científica. Este proceso se ha visto ampliamente enriquecido durante las últimas tres décadas con la aparición y auge de las revistas digitales disponibles en línea. Independientemente del soporte que las revistas utilicen, su acreditación ante las comunidades a las que sirven se sustenta en una serie de criterios que dan cuenta de su calidad y confiabilidad, tales como la revisión por pares, la conformación no endogámica de los cuerpos editoriales, la publicación de artículos de parte de autores ajenos a la institución editora de la revista y la variedad de idiomas de publicación. A esto se suma la presencia de la revista en bases de datos, especialmente en aquellas que aplican ejercicios de selección para la inclusión y difusión de sus contenidos.

En este documento se presenta un análisis de la calidad editorial de un grupo de revistas iberoamericanas especializadas en derecho y jurisprudencia, con base en el estudio de los datos proporcionados por el sistema de información regional iberoamericano Latindex (www.latindex.org). En aras de la confiabilidad del análisis, se emplea específicamente la información de las calificaciones que han recibido las revistas incluidas en el Catálogo Latindex. El valor del catálogo es generalmente reconocido por proporcionar información 
detallada sobre la calidad editorial de las revistas incluidas en él; ${ }^{1}$ al respecto, cabe mencionar que desde sus inicios el Catálogo Latindex ha sido empleado como referencia para la evaluación de las revistas científicas en varios países de la región. ${ }^{2}$

\section{Las bases de datos y la calidad de las revistas académicas}

La relación entre las bases de datos y las revistas académicas se remonta a las antiguas publicaciones de indización y resúmenes que surgieron con el propósito de proporcionar un acceso organizado y seleccionado a los contenidos de un creciente número de revistas especializadas que circulaban desde el siglo XVIII. La mayoría de las bases de datos existentes al día de hoy son producidas en Estados Unidos y países de Europa Occidental, y suelen tener un enfoque comercial, es decir, son compilaciones a las que se puede acceder únicamente a través de una suscripción. Si bien estas bases de datos, las de revistas especializadas, se definen ellas mismas como internacionales, un rasgo característico es que las publicaciones editadas en los países iberoamericanos suelen tener escasa presencia en ellas. Diversas razones se esgrimen para explicar o justificar tal situación: para unos, se debe a que nuestras revistas no son del interés de los lectores de otras latitudes; para otros, los criterios de inclusión están sesgados hacia el perfil de las revistas anglosajonas; otros más atribuyen esta escasa presencia de nuestras revistas a la falta de calidad de sus contenidos. El caso es que dichas bases de datos ofrecen en general información parcial e incompleta acerca de las revistas iberoamericanas, en todas las áreas del conocimiento. Esto coloca en seria desventaja a nuestra producción editorial y a los autores que la sostienen. Por un lado, los productos científicos con mayor visibilidad tienden a generar una mayor demanda e impacto en determinadas comunidades científicas; ${ }^{3}$ por otro lado, suele asociarse con la presencia de una revista en las grandes bases de datos comerciales un sello de prestigio mediante el empleo de los llamados indicadores bibliométricos- que incide en la

\footnotetext{
${ }^{1}$ Alperín, Juan Pablo; Babini, Dominique y Fischman Gustavo, Open Access Indicators. Assessing growth and use of OA resources from developing regions - the cases of Latin America and Africa, UNESCO, Clacso, PKP, Flacso, 2013; Chavarro Bohórquez, Diego Andrés, "Universalism and particularism: explaining the emergence and growth of regional journal indexing systems", tesis doctoral, Universidad de Sussex, 2017.

2 Rodríguez-Yunta, Luis, "Evaluación de las revistas españolas: pasos hacia un sistema que mida la apertura y la internacionalidad", Anuario ThinkEPI, núm. 9, 2015, pp.189-195; Flores, Ana María y Casado, Ana, "El sistema Latindex en Argentina", Ciência da informação, vol. 44, núm. 2, 2015, pp. 209-228.

${ }^{3}$ Garduño Oropeza, Gustavo y Zúñiga Roca, Ma. Fernanda, "La comunicación de la ciencia: génesis e implicaciones contextuales en revistas especializadas", Razón y Palabra, núm. 77, agosto-octubre, 2011.
} 
evaluación académica, tanto la individual como la institucional. Mucho se ha escrito y debatido sobre este tema. Para efectos del presente trabajo, recomendamos: San Francisco Declaration on Research Assessment (DORA) (2012) y la carta de adhesión a DORA, firmada por Latindex, Redalyc y Clacso.

Afortunadamente existen en Iberoamérica iniciativas como SciELO (www.scielo.org), Redalyc (https://www.redalyc.org/home.oa) y Redib (https://www.redib.org/), que manejan metodologías propias para valorar la calidad editorial de sus revistas, con una perspectiva que no se centra en los modelos estadísticos de medición generados por las bases de datos comerciales.

Una de estas iniciativas es el sistema Latindex, que desde su concepción buscó responder a dos cuestionamientos que habían sido largamente planteados en la comunidad académica latinoamericana: ¿cuántas revistas académicas y científicas existen en la región? y ¿cuál es su calidad? Para responder a la primera pregunta, Latindex inició en 1997 la construcción del directorio; y para la segunda, planteó la elaboración de un catálogo que comenzó a dar sus primeros resultados en 2002. Mientras que el directorio es una base de datos incluyente y exhaustiva, el catálogo es un producto selectivo, ya que incluye únicamente las revistas del directorio que cumplen con un umbral mínimo de requerimientos de calidad editorial. Para el presente estudio nos basamos exclusivamente en los registros incluidos en el catálogo, por ser este el que proporciona información cualitativa de actualidad sobre las revistas.

\section{Las revistas de derecho y jurisprudencia en Latindex}

Para que una revista sea registrada en el directorio debe cumplir con lo siguiente: 1 ) publicar contenidos de interés académico, 2) estar vigente al momento del registro, 3) tener al menos un número publicado y 4) contar con un registro ISSN o declarar que está en trámite. Bajo estos cuatro requerimientos, el Directorio Latindex da cuenta de 1,285 revistas clasificadas bajo el subtema derecho y jurisprudencia, de las cuales 1,157 aparecen como vigentes. 
El Catálogo Latindex, por su parte, demanda el cumplimiento de más requerimientos, por lo que no todas las revistas registradas en el directorio logran ingresar. Entre 2002 y 2017 operó la versión 1.0, que fue aplicada a más de 14,000 revistas impresas y electrónicas. El Catálogo 2.0, exclusivamente para revistas en línea, está vigente desde octubre de 2018, por lo que muchas revistas están aún en proceso de calificación. En esta versión, el catálogo contiene únicamente aquellas revistas que cumplen con al menos 30 de 38 características de calidad, incluidas siete obligatorias. Para mayor información sobre la metodología y características del Catálogo 2.0 de Latindex, consulte: https://www.latindex.org/latindex/meto2.

En el caso de las revistas de derecho y jurisprudencia, se identificaron 72 revistas ingresadas al Catálogo 2.0 hasta el 1 de abril de 2020 (véase Anexo). Estas revistas están editadas en los siguientes países: Argentina (9), Brasil (2), Chile (3), Costa Rica (1), Ecuador (3), España (34), México (12), Perú (2), Portugal (1), Uruguay (1) y Venezuela (1). Por ser la versión 2.0 de creación relativamente reciente, estos números no son representativos del total de revistas en línea editadas en cada país, sino más bien reflejan el ritmo de trabajo de cada centro nacional de acopio. Puesto que Latindex también registra revistas de contenido iberoamericanista -revistas editadas fuera de Iberoamérica pero cuyos contenidos están dedicados a estudiar asuntos iberoamericanos, latinoamericanos, hispanos o lusistas-, dos revistas de este perfil fueron incluidas en el estudio, una editada en Alemania y otra en Italia.

La mayoría de estas revistas (71\%) son editadas por instituciones educativas, principalmente universidades, mientras que el resto están repartidas, casi en igualdad, entre asociaciones profesionales, instituciones gubernamentales 0 editoriales privadas. En 26 de estas revistas el idioma predominante de publicación de los textos completos es el español, mientras que 20 publican en español o en inglés. Solamente dos revistas publican exclusivamente en inglés y una en portugués. El resto (23) son revistas que publican en varios idiomas (español, portugués, inglés, francés, alemán, italiano, catalán, vasco y gallego). 
En cuanto a su presencia en los dos índices comerciales más influyentes: Web of Science (WoS) y Scopus, encontramos que 15 de las 72 revistas están indizadas en WoS, 9 en Scopus y 5 en ambas. En el caso de WoS, todas las indizaciones corresponden a Emerging Source Citation Index (ESCI), que es una de las cuatro bases de datos consideradas parte de la colección núcleo de la WoS. Hay que hacer notar que ESCI se conforma por las revistas que están en proceso de evaluación e ingreso en las otras bases de datos principales (Science Citation Index Expanded, Social Sciences Citation Index y Arts \& Humanities Citation Index). La inclusión en ESCI permite, desde el proceso de evaluación, conocer si las revistas reciben citas y quién las cita.

\section{La calidad de las revistas de derecho y jurisprudencia}

La metodología del Catálogo 2.0 es aplicada exclusivamente a revistas digitales que poseen un sitio web propio o institucional. Para ser calificadas, deben tener al menos dos años de antigüedad y ser de acceso abierto. La calificación se basa en las evidencias encontradas en los sitios web, ya que no se utiliza ninguna otra fuente de información para comprobar su cumplimiento. Las 38 características están repartidas en cinco grupos, y por cada característica cumplida se asigna un punto. Para obtener la categoría Catálogo Latindex las revistas han de cumplir con $80 \%$ de las características.

El grupo de características obligatorias, observado al 100\% por las revistas del estudio, está enfocado en comprobar que se cumplan requerimientos básicos para cualquier revista que se precie de ser académica. Las revistas deben demostrar, entre otras cosas, que aplican un sistema de arbitraje, describiéndolo en cada uno de sus pasos e informando del uso de evaluadores externos a la institución editora; asimismo, las instrucciones a los autores deben estar siempre disponibles en el sitio web y demostrar que la revista ha generado nuevos contenidos durante los últimos dos años consecutivos. Este primer filtro permite avanzar en la calificación de las 
restantes 31 características, con la confianza de que la metodología se está aplicando a una revista que observa tanto normas básicas como buenas prácticas editoriales.

El segundo grupo contempla características de presentación que informan sobre el apego a normatividad editorial de carácter internacional. En este grupo, las fechas de recepción y aceptación de originales y el membrete bibliográfico en cada artículo fueron los de menor cumplimiento, apareciendo en 63 y 66 de las revistas, respectivamente. En cambio, la mención de periodicidad y la afiliación institucional de los miembros de los cuerpos editoriales fueron cumplidas por la totalidad de las revistas estudiadas.

El tercer grupo refleja la gestión y las políticas editoriales establecidas para cada revista. Aquí los niveles de cumplimiento fueron más variados; los de menor observancia fueron las políticas para detección de plagio (presentes en sólo 42 revistas), la adopción de códigos de ética (62), la apertura editorial (63) y el cumplimiento de la periodicidad declarada, que debe darse al inicio del período de publicación (64).

El cuarto grupo, denominado características de contenido, califica la presencia de elementos normativos que reflejan también buenas prácticas editoriales en la estructura de un artículo científico, tales como la presencia de resúmenes y palabras clave, la exigencia de originalidad en los artículos postulados y al menos un $40 \%$ de contenido original en cada número, entre otras. Después del grupo de características básicas, es el que presenta el mayor grado de cumplimiento general, puesto que contiene características que las revistas académicas han adoptado desde hace décadas y que han sido fácilmente trasladadas de las versiones impresas a las digitales. Sin embargo, llama la atención que en las instrucciones para la elaboración de las referencias bibliográficas se tiende a ignorar el uso de normas reconocidas internacionalmente, y esto es un requerimiento para cumplir el criterio respectivo. 
Finalmente, el quinto grupo reúne todas las características que permiten identificar una buena revista digital. Al igual que lo encontrado en otros estudios similares, ${ }^{4}$ este grupo resulta ser el que más dificultades presenta para su cumplimiento. Tomando en cuenta esta situación, revisamos los resultados de cada una de sus ocho características.

La presencia de motores de búsqueda en los sitios web resultó ser la característica mejor cumplida, al estar presente en 70 de las 72 revistas; buena parte de este resultado se debe a que, en su mayoría, los sitios usan la plataforma de gestión Open Journal System (OJS), que facilita la integración de varias de las características contempladas por el Catálogo Latindex.

En orden decreciente, sigue la presencia en 62 revistas de servicios de valor agregado; estos servicios, en su mayoría, son: facilidades para lectores con discapacidad visual a través de herramientas que aumentan el tamaño de la fuente, disponibilidad del sitio web en más de un idioma, disponer de un texto sobre el historial de la revista u ofrecer la cita de cada artículo publicado en diferentes formatos de citación.

El uso de identificadores uniformes de recursos, particularmente del Digital Object Identifier (DOI), está presente en $65 \%$ de las revistas (47), mientras que los servicios de interactividad los encontramos en 44 de las publicaciones (en la mayoría de los casos se trata de presencia de las revistas en diversas redes sociales).

Con una ocurrencia por debajo de $50 \%$ de los títulos, encontramos el uso de estadísticas (34 revistas), las políticas de preservación digital (28 revistas) y el uso de diferentes formatos para la lectura de los textos completos (24 revistas). En este último caso se identificó la utilización muy extendida del PDF como único formato para

\footnotetext{
${ }^{4}$ Reyna Espinosa, Felipe Rafael y Alonso Gamboa, José Octavio, "Las revistas digitales de bibliotecología y ciencia de la información frente a los nuevos criterios de calidad de Latindex", Ibersid, vol. 11, núm. 2, julio-diciembre, 2017, pp. 59-64.
} 
el despliegue de los textos, en detrimento de otros como HTML O XML, que brindan una riqueza de posibilidades para la búsqueda y recuperación de información en revistas digitales.

Los resultados del estudio dan cuenta de un buen trabajo editorial para incorporar aspectos normativos y técnicos que facilitan el proceso de comunicación científica. Al igual que en revistas de otras disciplinas, las publicaciones aquí analizadas muestran una adecuada apropiación de elementos muy depurados en el trabajo editorial de carácter científico. Al igual que lo encontrado en revistas de otras disciplinas, las características de tipo técnico, específicamente las referidas a la calidad digital de los sitios web, presentan diferentes grados de integración que habrán de mejorarse; para lograr la plena integración de características digitales será indispensable la capacitación permanente de los equipos editoriales de apoyo.

\section{Reflexiones sobre el futuro de las revistas}

El panorama que ofrece este conjunto - aún limitado- de revistas iberoamericanas de derecho y jurisprudencia es alentador puesto que pone de manifiesto la vocación constante de mejora por parte de los editores, así como el compromiso de instituciones editoras y autores por apoyar y mantener vigentes sus revistas como vehículos válidos de comunicación del trabajo académico. Habida cuenta de que ni la labor editorial ni las publicaciones en revistas de factura iberoamericana suelen ser especialmente valoradas por los sistemas de evaluación, estos esfuerzos resultan tanto más significativos.

Una ojeada a los contenidos de las revistas analizadas permite entender al menos en parte las razones que subyacen a este sentido de compromiso. Los temas objeto de investigación, la actualidad y relevancia de la problemática abordada y la forma de abordarla, las preguntas que se plantean los autores y el carácter crítico de sus análisis hace ver que estas revistas son no sólo pertinentes, sino necesarias, tanto aquellas especializadas que publican nuevos resultados como las orientadas a la enseñanza o a la difusión del conocimiento. El que para los grandes índices 
comerciales puedan no ser de interés, por no aportar a su negocio, no les resta validez, si bien, claro está, su inclusión en ellos ayuda a su visibilidad y prestigio.

Es importante, por lo anterior, que estas revistas $-\mathrm{y}$ otras que se espera ingresen próximamente al Catálogo 2.0- no sólo aseguren su vigencia y su relevancia, sino que se coloquen - si no lo están ya - a la vanguardia en las investigaciones del área jurídica de su competencia, manteniendo el espíritu crítico y trazando cada una de ellas su propio camino, Por otro lado, más allá de cumplir con estándares de calidad editorial, es imperativo para las revistas estar atentas a las nuevas oportunidades que brinda la comunicación en línea. Además de los servicios de valor agregado mencionados en la sección 4, día con día surgen nuevas herramientas y modalidades de comunicación que van transformando a la revista en cuanto que permiten formas más expeditas de dar a conocer resultados y facilitan el diálogo fluido y abierto entre colegas, autores y lectores.

\section{Bibliografía}

Alperín, Juan Pablo; Babini, Dominique y Fischman Gustavo, Open Access Indicators. Assessing growth and use of $O A$ resources from developing regions - the cases of Latin America and Africa, UNESCO, Clacso, PKP, Flacso, 2013 [consultado el 11 de abril de 2020]. Disponible en: https://www.researchgate.net/publication/308709678 Open Access indicators Assessing growth and use of $\mathrm{OA}$ resources from developing regions the $c$ ases of Latin America and Africa.

Carta de adhesión a la Declaración de San Francisco sobre la evaluación de la investigación (DORA), México, 15 de enero de 2019 [consultado el 11 de abril de 2020]. Disponible en: https://sfdora.org/read/es/.

Centro de Documentación Científica y Técnica de México, Guía de Publicaciones Periódicas Científicas y Técnicas de América Latina: una lista anotada, México, Unión Panamericana, 1962.

Chavarro Bohórquez, Diego Andrés, Universalism and particularism: explaining the emergence and growth of regional journal indexing systems, tesis doctoral, Universidad de Sussex, 2017 [consultado el 11 de abril de 2020]. Disponible en: http://sro.sussex.ac.uk/id/eprint/66409/.

Flores, Ana María y Casado, Ana, "El sistema Latindex en Argentina", Ciência da informação, vol. 44, núm. 2, 2015, pp. 209-228 [consultado el 12 de abril de 2020]. Disponible en: http://revista.ibict.br/ciinf/article/view/1860.

Garduño Oropeza, Gustavo y Zúñiga Roca, Ma. Fernanda, "La comunicación de la ciencia: génesis e implicaciones contextuales en revistas especializadas", Razón y palabra, núm. 77, agosto-octubre, 2011 [consultado el 9 de abril de 2020]. 
Disponible

en: http://www.razonypalabra.org.mx/varia/77\%203a\%20parte/47_GardunoZuniga_V 77.pdf.

Hicks, Diana; Wouters, Paul; Waltman, Ludo; De Rijcke, Sarah y Ràfols, Ismael, "Bibliometrics: the Leiden manifesto for research metrics", Nature, vol. 520, núm. 7548, pp. 429-31, 2015 [consultado el 12 de abril de 2020]. Disponible en: https://doi.org/10.1038/520429a.

Panier, D. F., "Informe sobre la situación actual de las revistas científicas y técnicas latinoamericanas", grupo de trabajo para la selección de revistas científicas latinoamericanas, San Juan, Puerto Rico, abril 28-mayo 1, 1964. Centro de Cooperación Científica de la UNESCo para América Latina, Montevideo, Uruguay, julio 1964.

Ràfols, Ismael; Molas-Gallart, Jordi; Chavarro, Diego Andrés y Robinson García, Nicolás, "On the dominance of quantitative evaluation in 'peripheral' countries: auditing research with technologies of distance", SSRN Electronic Journal, mayo 28 2016 [consultado el 11 de abril de 2020]. Disponible en: http://dx.doi.org/10.2139/ssrn.2818335.

Reyna Espinosa, Felipe Rafael y Alonso Gamboa, José Octavio, "Las revistas digitales de bibliotecología y ciencia de la información frente a los nuevos criterios de calidad de Latindex", Ibersid, vol. 11, núm. 2, julio-diciembre, 2017, pp. 59-64 [consultado el 12 de abril de 2020]. Disponible en: https://www.ibersid.eu/ojs/index.php/ibersid/article/view/4452/3892.

Rodríguez-Yunta, Luis, "Evaluación de las revistas españolas: pasos hacia un sistema que mida la apertura y la internacionalidad", Anuario ThinkEPI, núm. 9, pp.189195, 2015 [disponible en: https://digital.csic.es/bitstream/10261/125672/1/35692112454-1-PB.pdf.

"San Francisco Declaration on Research Assessment (DORA)", 2012 [consultado el 11 de abril de 2020]. Disponible en: https://sfdora.org/. Versión en español disponible en: https://revistaius.com/index.php/ius/pages/view/dora.

Vessuri, Hebe; Guédon, Jean-Claude y Cetto, Ana María, "Excellence or quality? Impact of the current competition regime on science and scientific publishing in Latin America and its implications for development", Current Sociology, vol. 62, núm. 5, 2014, pp. 647-65 [consultado el 11 de abril de 2020]. Disponible en: https://doi.org/10.1177/0011392113512839. 